\title{
MonoPhy: A simple R package to find and visualize monophyly issues
}

Orlando Schwery, Brian C O'Meara

Background. The monophyly of taxa is an important attribute of a phylogenetic tree. A lack of it may hint at shortcomings of either the tree or the current taxonomy, or can indicate cases of incomplete lineage sorting or horizontal gene transfer. Whichever is the reason, a lack of monophyly can misguide subsequent analyses. While monophyly is conceptually simple, it is manually tedious and time consuming to assess on modern phylogenies of hundreds to thousands of species. Results. The R package MonoPhy allows assessment and exploration of monophyly of taxa in a phylogeny. It can assess the monophyly of genera using the phylogeny only, and with an additional input file any other desired higher order taxa or unranked groups can be checked as well. Conclusion. Summary tables, easily subsettable results and several visualization options allow quick and convenient exploration of monophyly issues, thus making MonoPhy a valuable tool for any researcher working with phylogenies. 


\section{MonoPhy: A simple R package to find and visualize monophyly}

2 issues

3 Orlando Schwery and Brian C. O'Meara

4 Department of Ecology \& Evolutionary Biology, University of Tennessee, Knoxville, TN 37996-1610, USA

5

6 Corresponding Author:

7 Orlando Schwery

8 425a Hesler, University of Tennessee, Knoxville, TN 37996-1610, USA

9 Email address: oschwery@vols.utk.edu 


\section{Abstract}

12 Background. The monophyly of taxa is an important attribute of a phylogenetic tree. A lack of it 13 may hint at shortcomings of either the tree or the current taxonomy, or can indicate cases of 14 incomplete lineage sorting or horizontal gene transfer. Whichever is the reason, a lack of 15 monophyly can misguide subsequent analyses. While monophyly is conceptually simple, it is 16 manually tedious and time consuming to assess on modern phylogenies of hundreds to thousands 17 of species.

18 Results. The R package MonoPhy allows assessment and exploration of monophyly of taxa in a phylogeny. It can assess the monophyly of genera using the phylogeny only, and with an additional input file any other desired higher order taxa or unranked groups can be checked as well.

Conclusion. Summary tables, easily subsettable results and several visualization options allow quick and convenient exploration of monophyly issues, thus making MonoPhy a valuable tool for any researcher working with phylogenies.

\section{Introduction}

26

27

28

29

30

31

32

33

34

35

36

37

38

39

40

41

42

43

44

45

46

47

Phylogenetic trees are undoubtedly crucial for most research in ecology or evolutionary biology. Whether one is studying trait evolution (e.g. Coddington 1988; Donoghue 1989), diversification (e.g. Gilinsky \& Good 1991; Hey 1992), phylogeography (Avise et al. 1987), or simply relatedness within a group (e.g. Czelusniak et al. 1982; Shochat \& Dessauer 1981; Sibley \& Ahlquist 1981), bifurcating trees representing hierarchically nested relationships are central to the analysis. Exactly because phylogenies are so fundamental to the inferences we make, we need tools that enable us to examine how reconstructed relationships compare with existing assumptions, particularly taxonomy. We have computational approaches to estimate confidence for parts of a phylogeny (Felsenstein 1985; Larget \& Simon 1999) or measuring distance between two phylogenies (Robinson 1971), but assessing agreement of a new phylogeny with existing taxonomy is often done manually. This does not scale to modern phylogenies of hundreds to thousands of taxa. Modern taxonomy seeks to name clades: an ancestor and all of its descendants (the descendants thus form a monophyletic group). Discrepancies between the new phylogenetic hypothesis and the current taxonomic classification may indicate that the phylogeny is wrong or poorly resolved. Alternatively, a well-supported phylogeny that conflicts with currently recognized groups might suggest that the taxonomy should be reformed. To identify such discrepancies, one can simply assess whether the established taxa are monophyletic. A lack of group monophyly however, can also be an indicator for conflict between gene trees and the species tree, which may be a result of incomplete lineage sorting or horizontal gene transfer. In any case, monophyly issues in a phylogeny suggest a potential error that can affect downstream analysis and inference. For example, it will mislead ancestral trait or area reconstruction or introduce false signals when assigning unsampled diversity for 
48

49

50

51

52

53

54

55

56

57

58

59

60

61

62

63

64

65

66

67

68

69

70

71

72

73

74

75

76

77

78

79

80

81

82

83

84

85

diversification analyses (e.g. in diversitree (FitzJohn 2012) or BAMM (Rabosky 2014)). In general, a lack of monophyly can blur patterns we might see in the data otherwise.

As this problem is by no means new, approaches to solve it have been developed earlier, particularly for large scale sequencing projects in bacteria and archaea, for which taxonomic issues are notoriously challenging. The program GRUNT (Dalevi et al. 2007) uses a tip to root walk approach to group, regroup, and name clades according to certain user defined criteria. The subsequently developed 'taxonomy to tree' approach (McDonald et al. 2012) matches existing taxonomic levels onto newly generated trees, allowing classification of unidentified sequences and proposal of changes to the taxonomic nomenclature based on tree topology. Finally, Matsen \& Gallagher (2012) have developed algorithms that find mismatches between taxonomy and phylogeny using a convex subcoloring approach.

The new tool presented here, the R package MonoPhy, is a quick and user-friendly method for assessing monophyly of taxa in a given phylogeny. While the $\mathrm{R}$ package ape (Paradis et al. 2004) already contains the helpful function is .monophyletic, which also enables testing for monophyly, the functionality of MonoPhy is much broader. Apart from assessing monophyly for all groups and focal taxonomic levels in a tree at once, MonoPhy is also not limited to providing a simple 'yes-or-no' output, but rather enables the user to explore underlying causes of nonmonophyly. In the following, we outline the structure and usage of the package and provide examples to demonstrate its functionality. For a more usage-focused and application-oriented treatment, one should refer to the tutorial vignette (vignette ("MonoPhyVignette")), which contains stepwise instructions for the different functions and their options. For any other package details consult the documentation (help ("MonoPhy") ).

\section{Description}

The package MonoPhy is written in R (R Development Core Team 2014, http://www.Rproject.org/), an increasingly important language for evolutionary biology. It builds on the existing packages ape (Paradis et al. 2004), phytools (Revell 2012), phangorn (Schliep 2011), RColorBrewer (Neuwirth 2014) and taxize (Chamberlain \& Szocs 2013). A list of the currently implemented commands is given in Table 1. Note that in the code and this paper, we distinguish between tips, the organisms at the tip of the tree, and higher order taxa. Functions with 'taxa' only return information about higher order taxa, not tips. The main function -

Assessmonophyly - evaluates the monophyly of each higher order taxon by identifying the most recent common ancestor (MRCA) of a collection of tips (e.g. all species in a genus), and then returning all descendants of this node. The taxon is monophyletic if the number of its members (tips) equals the number of descendants of its MRCA. If there are more descendants than taxon members, the function will identify and list the tips that do not belong to the focal taxon and we then call these tips 'intruders'. Accordingly, we will further refer to the taxa whose monophyly was disrupted by these 'intruders' as 'intruded'. Note that if two taxa are reciprocally disrupting each other's monophyly, certain tips of intruded taxa will often be intruders 
86 themselves: if the phylogeny is $((\mathrm{A} 1, \mathrm{~B} 1),(\mathrm{A} 2, \mathrm{~B} 2))$, where $\mathrm{A}$ and $\mathrm{B}$ are genera, it's not clear if the

87 A tips are intruding in $\mathrm{B}$ or the $\mathrm{B}$ tips are intruding in $\mathrm{A}$.

88

Table 1: Functions of the package MonoPhy.

Function name

Assessmonophyly

GetAncNodes

GetIntruderTaxa

GetIntruderTips

GetoutlierTaxa

GetoutlierTips

GetResultmonophyly

Get SummaryMonophyly

PlotMonophyly

\section{Description}

Runs the main analysis to assess monophyly of groups on a tree Returns MRCA nodes for taxa.

Returns lists of taxa that cause monophyly issues for another taxon.

Returns lists of tips that cause monophyly issues for a taxon.

Returns lists of taxa that have monophyly issues due to outliers.

Returns lists of tips that cause monophyly issues for their taxon by being outliers.

Returns an extended table of the results

Returns a summary table of the results

Allows several visualizations of the result.
90

91

92

93

94

95

96

97

98

99

100

101

102

103

104

105

106

107

108

109

110

111

112

Biologically, identifying a few intruders may suggest that the definition of a group should be expanded; observing some group members in very different parts of the tree than the rest of their taxon may instead suggest that these individuals were misidentified, that their placement is the result of contaminated sequences or due to horizontal gene transfer between members of two remote clades. Moreover, the approach as described above would suggest that the clades that are intruded by the outlier tips would in turn be intruders to the taxon the outliers belong to, which intuitively would not make sense. We thus implemented an option to specify a cutoff value, which defines the minimal proportion of tips among the descendants of a taxon's MRCA that are labeled as being actual members of that taxon. If a given group falls below this value, the function will find the 'core clade' (a subclade for which the proportion matches or exceeds the cutoff value) by moving tipward, always following the descendant node with the greater number of tips in the focal taxon (absolute, relative if tied), and at each step evaluating the subtree rooted at that node to see if it exceeds the cutoff value. Once such a subtree is found, it is then called the 'core clade', and taxon members outside this clade are then called 'outliers'. As there is no objective criterion to decide at what point individuals should be considered outliers, a reasonable cutoff value must be chosen by the user.

If the tree's tip labels are in the format 'Genus_speciesepithet', the genus names will be extracted and used as taxon assignments for the tips. If the tip labels are in another format, or other taxonomic levels should be tested, taxon names can be assigned to the tips using an input file. To avoid having to manually compose a taxonomy file for a taxon-rich phylogeny, MonoPhy can automatically download desired taxonomic levels from ITIS or NCBI using taxize (Chamberlain \& Szocs 2013). 
113 All inference results are stored in a solution object, from which the other functions can extract 114 information (e.g. summary tables, intruder and outlier lists) for one or more higher-level taxa of 115 interest. PlotMonophyly reconstructs and plots the monophyly state of the tips using phytools 116 (Revell 2012). Apart from the basic monophyly plot (Fig.1), branches can be coloured according 117 to taxonomic groups or to highlight intruders and outliers. Monophyletic groups can be collapsed 118 and plots can be saved directly to PDF to facilitate the visualization of large trees.

119 It is important to remember that the results produced by the package are merely the product of 120 the used phylogeny and the available taxonomic information. It thus only makes the mismatches 121 between those accessible, but does not reveal any more than that. The decision of whether the 122 result suggests problems in the phylogeny or the taxonomy, whether a tip should be considered a 123 rogue taxon and be removed or whether gene tree - species tree conflicts should be investigated, 124 is entirely up to the user's judgment.

125 MonoPhy is available through CRAN (https://cran.r-project.org/package=MonoPhy/) and is 126 developed on GitHub (https://github.com/oschwery/MonoPhy). Intended extensions and fixes 127 can be seen in the issues list of the package's GitHub page. Among the planned extensions of the 128 package are: multiple trees, displaying the result for specific subtrees, proposing monophyletic 129 subgroups, enabling formal tests for monophyly (incorporating clade support) and providing 130 increased plot customizability.

\section{Examples}

132 Our first example makes use of the example files contained in the package. They come from a 133 phylogeny of the plant family Ericaceae (Schwery et al. (2015) pruned to 77 species; original 134 data see Schwery et al. (2014)) and two taxon files assigning tribes and subfamilies to the tips (in 135 both files, errors have been introduced for demonstration purposes; see code and output for both 136 examples in Supplementary Data). Running the main analysis command AssessMonophyly 137 on genus level (i.e. tree only) and tribe level (i.e. tree plus taxonomy file) using standard settings 138 took 0.045 and 0.093 seconds respectively on a MacBook Pro with $2.4 \mathrm{GHz}$ Intel Core i5 and 139 8GB Ram. We could now use the remaining commands to extract the information of interest 140 from the saved output object (e.g. summary tables, lists of problem taxa, etc.). The basic 141 monophyly plot for the genus level analysis is displayed for a subclade of the tree in Figure 1 142 (the figure of the full tree is shown in Fig. S1). 
143 For the second example, we demonstrate the package's performance on a tree of 31,749

144 species of Embriophyta (Zanne et al. 2014; data see Zanne et al. 2013), using an outlier-cutoff of 1450.9 this time. Just checking monophyly for genera took 1.78 hours, but revealed that $22 \%$ of

146 genera on the tree are not monophyletic, while around half of all genera are only represented by 147 one species each. Furthermore, we can see that the largest monophyletic genus is Iris (139 tips), 148 that Justicia had the most intruders (13 tips) and that Acacia produced the most outliers (99 tips). 149 Finally, with 2337 other tips as descendants of their MRCA, the 3 species of Aldina are most 150 spread throughout the tree.

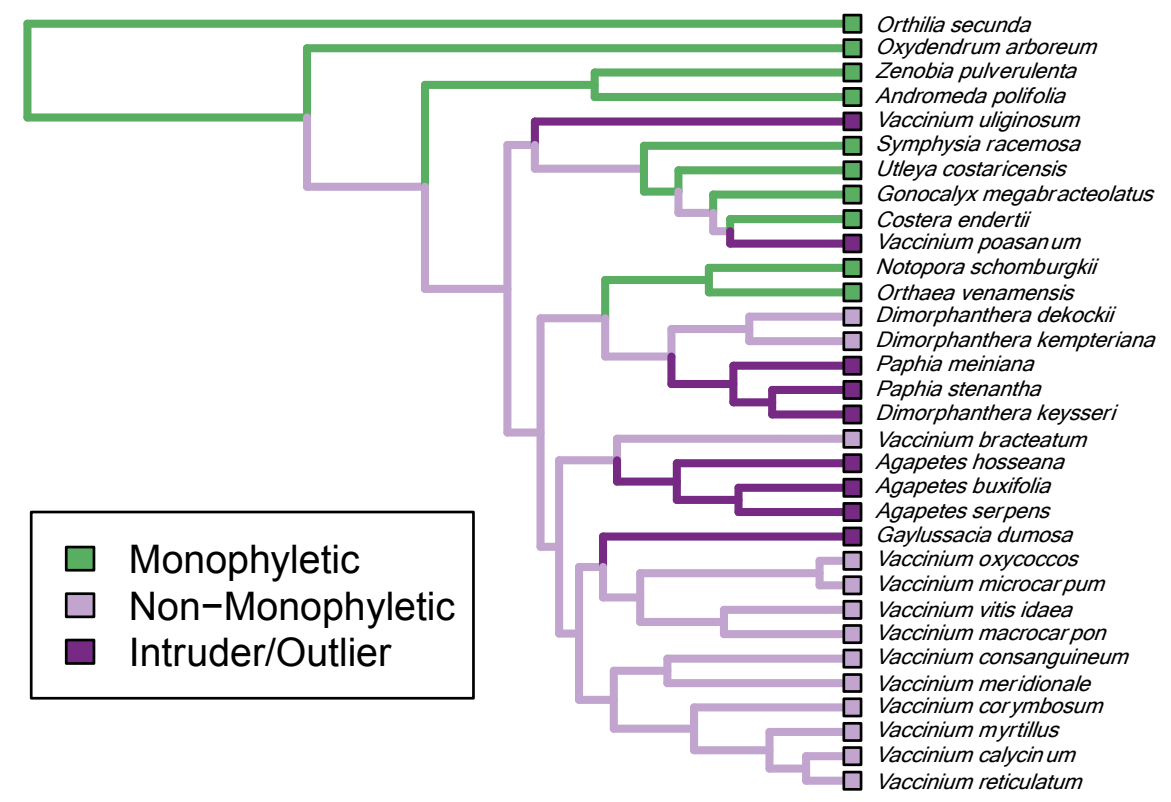

151 Fig. 1. Monophyly plot of the genera of Ericaceae. Close-up on subfamily Vaccinioideae only. Branches of the 152 tree coloured according to monophyly status. We can see that Vaccinium has two outliers and that its intruders are 153 Paphia, Dimorphanthera, Agapetes and Gaylussacia.

\section{Citation}

155 Researchers using MonoPhy in a published paper should cite this article and indicate the 156 used version of the package. The citation information for the current package version can 157 be obtained using citation ("MonoPhy").

\section{Acknowledgements}

159 We want to thank the members of the O'Meara lab for helpful discussions, Frederik Matsen 160 IV and two anonymous reviewers for well-considered criticism to improve the manuscript, 161 Brian Looney and Sam Borstein for beta testing, and the members of the Tank lab, Arne 162 Mooers, Karen Cranston, Bruce Cochrane and Daniel Gates for great ideas on increasing the 163 usefulness of this package. 
164

165

166

167

168

169

170

171

172

173

174

175

176

177

178

179

180

181

182

183

184

185

186

187

188

189

190

191

192

193

194

195

196

197

198

199

200

201

202

203

204

205

206

\section{References}

Avise JC, Arnold J, Ball RM, Bermingham E, Lamb T, Neigel JE, Reeb CA, and Saunders NC. 1987. Intraspecific Phylogeography - The Mitochondrial-DNA Bridge Between Population-Genetics and Systematics. Annual Review of Ecology and Systematics 18:489-522.

Chamberlain SA, and Szocs E. 2013. taxize: taxonomic search and retrieval in R. F1000Research 2:191-191.

Coddington JA. 1988. Cladistic Tests Of Adaptational Hypotheses. Cladistics-the International Journal of the Willi Hennig Society 4:3-22.

Czelusniak J, Goodman M, Hewettemmett D, Weiss ML, Venta PJ, and Tashian RE. 1982. Phylogenetic Origins and Adaptive Evolution of Avian and Mammalian Hemoglobin Genes. Nature 298:297-300.

Dalevi D, DeSantis TZ, Fredslund J, Andersen GL, Markowitz VM, and Hugenholtz P. 2007. Automated group assignment in large phylogenetic trees using GRUNT: GRouping, ungrouping, naming tool. Bmc Bioinformatics 8.

Donoghue MJ. 1989. Phylogenies and the Analysis of Evolutionary Sequences, with Examples from Seed Plants. Evolution 43:1137-1156.

Felsenstein J. 1985. Confidence Limits on Phylogenies: An Approach Using the Bootstrap. Evolution 39:783-791.

FitzJohn RG. 2012. Diversitree: comparative phylogenetic analyses of diversification in R. Methods in Ecology and Evolution 3:1084-1092.

Gilinsky NL, and Good IJ. 1991. Probabilities of Origination, Persistence, and Extinction of Families of Marine Invertebrate Life. Paleobiology 17:145-166.

Hey J. 1992. Using Phylogenetic Trees to Study Speciation and Extinction. Evolution 46:627640.

Larget B, and Simon DL. 1999. Markov chain Monte Carlo algorithms for the Bayesian analysis of phylogenetic trees. Molecular Biology and Evolution 16:750-759.

Matsen FA, and Gallagher A. 2012. Reconciling taxonomy and phylogenetic inference: formalism and algorithms for describing discord and inferring taxonomic roots. Algorithms for Molecular Biology 7.

McDonald D, Price MN, Goodrich J, Nawrocki EP, DeSantis TZ, Probst A, Andersen GL, Knight R, and Hugenholtz P. 2012. An improved Greengenes taxonomy with explicit ranks for ecological and evolutionary analyses of bacteria and archaea. Isme Journal 6:610-618.

Neuwirth E. 2014. RColorBrewer: ColorBrewer Palettes. R package version 1.1-2. ed.

Paradis E, Claude J, and Strimmer K. 2004. APE: Analyses of Phylogenetics and Evolution in R language. Bioinformatics 20:289-290.

R Development Core Team. 2014. R: A language and environment for statistical computing. Vienna, Austria: R Foundation for Statistical Computing.

Rabosky DL. 2014. Automatic detection of key innovations, rate shifts, and diversitydependence on phylogenetic trees. Plos One 9:e89543.

Revell LJ. 2012. phytools: an R package for phylogenetic comparative biology (and other things). Methods in Ecology and Evolution 3:217-223. 
207

208

209

210

211

212

213

214

215

216

217

218

219

220

221

222

223

224

225

226

227

228

229

230

231
Robinson DF. 1971. Comparison of labeled trees with valency three. Journal of Combinatorial Theory, Series B 11:105-119.

Schliep KP. 2011. phangorn: phylogenetic analysis in R. Bioinformatics 27:592-593.

Schwery O, Onstein RE, Bouchenak-Khelladi Y, Xing Y, Carter RJ, and Linder HP. 2014. Data from: As old as the mountains: the radiations of the Ericaceae. Dryad Data Repository.

Schwery O, Onstein RE, Bouchenak-Khelladi Y, Xing Y, Carter RJ, and Linder HP. 2015. As old as the mountains: the radiations of the Ericaceae. New Phytologist 207:355-367.

Shochat D, and Dessauer HC. 1981. Comparative Immunological Study of Albumins of Anolis Lizards of the Caribbean Islands. Comparative Biochemistry and Physiology aPhysiology 68:67-73.

Sibley CG, and Ahlquist JE. 1981. The phylogeny and relationships of the ratite birds as indicated by DNA-DNA hybridization.

Zanne AE, Tank DC, Cornwell WK, Eastman JM, Smith SA, FitzJohn RG, McGlinn DJ, O'Meara BC, Moles AT, Reich PB, Royer DL, Soltis DE, Stevens PF, Westoby M, Wright IJ, Aarssen L, Bertin RI, Calaminus A, Govaerts R, Hemmings F, Leishman MR, Oleksyn J, Soltis PS, Swenson NG, Warman L, and Beaulieu JM. 2014. Three keys to the radiation of angiosperms into freezing environments. Nature 506:89-+.

Zanne AE, Tank DC, Cornwell WK, Eastman JM, Smith SA, FitzJohn RG, McGlinn DJ, O'Meara BC, Moles AT, Reich PB, Royer DL, Soltis DE, Stevens PF, Westoby M, Wright IJ, Aarssen L, Bertin RI, Calaminus A, Govaerts R, Hemmings F, Leishman MR, Oleksyn J, Soltis PS, Swenson NG, Warman L, Beaulieu JM, and Ordonez A. 2013. Data from: Three keys to the radiation of angiosperms into freezing environments. Dryad Data Repository. 